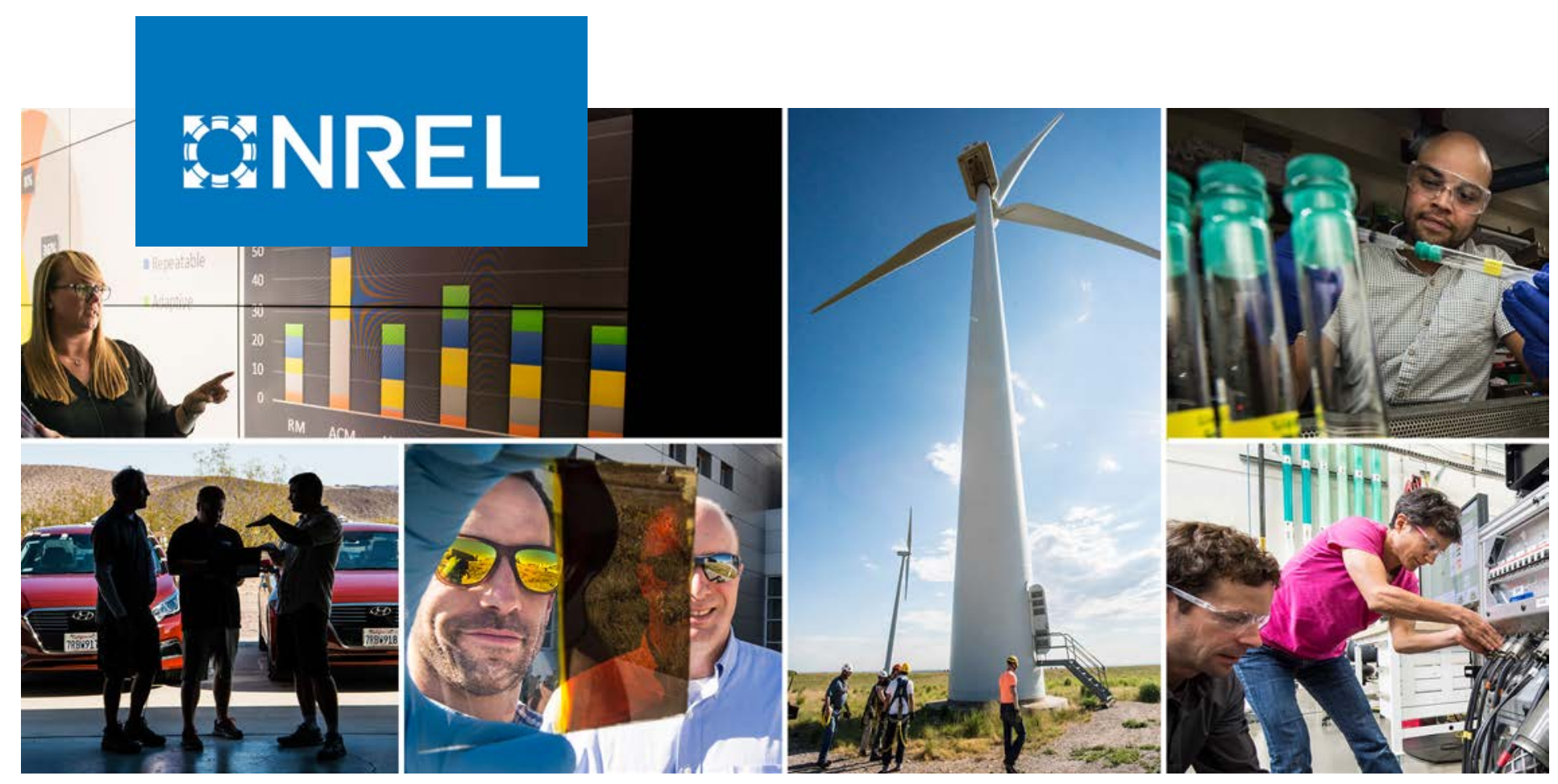

\title{
Blade-Resolved, Single-Turbine Simulations Under Atmospheric Flow
}

Michael Lawson, ${ }^{1}$ Jeremy Melvin, ${ }^{2}$ Shreyas Ananthan, ${ }^{1}$ Kenny Gruchalla, ${ }^{1}$ Jon Rood, ${ }^{1}$ and Michael Sprague ${ }^{1}$

1 National Renewable Energy Laboratory

2 University of Texas at Austin

NREL is a national laboratory of the U.S. Department of Energy Office of Energy Efficiency \& Renewable Energy

Operated by the Alliance for Sustainable Energy, LLC

This report is available at no cost from the National Renewable Energy Laboratory (NREL) at www.nrel.gov/publications.
Technical Report

NREL/TP-5000-72760

January 2019 


\title{
GHREL
}

\section{Blade-Resolved, Single-Turbine Simulations Under Atmospheric Flow}

\author{
Michael Lawson, ${ }^{1}$ Jeremy Melvin, ${ }^{2}$ Shreyas Ananthan, ${ }^{1}$ \\ Kenny Gruchalla, ${ }^{1}$ Jon Rood, ${ }^{1}$ and Michael Sprague ${ }^{1}$
}

\section{National Renewable Energy Laboratory}

2 University of Texas at Austin

\section{Suggested Citation}

Lawson, Michael, Jeremy Melvin, Shreyas Ananthan, Kenny Gruchalla, Jon Rood, and Michael Sprague. 2018. Blade-Resolved, Single-Turbine Simulations Under Atmospheric Flow. Golden, CO: National Renewable Energy Laboratory. NREL/TP-5000-72760.

https://www.nrel.gov/docs/fy19osti/72760.pdf.

NREL is a national laboratory of the U.S. Department of Energy Office of Energy Efficiency \& Renewable Energy Operated by the Alliance for Sustainable Energy, LLC

This report is available at no cost from the National Renewable Energy Laboratory (NREL) at www.nrel.gov/publications.

Contract No. DE-AC36-08GO28308
Technical Report NREL/TP-5000-72760 January 2019

National Renewable Energy Laboratory 15013 Denver West Parkway Golden, CO 80401 303-275-3000 • www.nrel.gov 


\section{NOTICE}

This work was authored in part by the National Renewable Energy Laboratory, operated by Alliance for Sustainable Energy, LLC, for the U.S. Department of Energy (DOE) under Contract No. DE-AC36-08GO28308. Funding provided by the Exascale Computing Project (17-SC-20-SC), a joint project of the U.S. Department of Energy's Office of Science and National Nuclear Security Administration, responsible for delivering a capable exascale ecosystem, including software, applications, and hardware technology, to support the nation's exascale computing imperative. The views expressed herein do not necessarily represent the views of the DOE or the U.S. Government.

This report is available at no cost from the National Renewable Energy Laboratory (NREL) at www.nrel.gov/publications.

U.S. Department of Energy (DOE) reports produced after 1991 and a growing number of pre-1991 documents are available free via www.OSTI.gov.

Cover Photos by Dennis Schroeder: (clockwise, left to right) NREL 51934, NREL 45897, NREL 42160, NREL 45891, NREL 48097, NREL 46526.

NREL prints on paper that contains recycled content. 


\section{Executive Summary}

The goal of the ExaWind project is to enable predictive simulations of wind farms comprised of many megawattscale turbines situated in complex terrain. Predictive simulations will require computational fluid dynamics (CFD) simulations for which the mesh resolves the geometry of the turbines and captures the rotation and large deflections of blades. Whereas such simulations for a single turbine are arguably petascale class, multi-turbine wind farm simulations will require exascale-class resources.

The primary code in the ExaWind project is Nalu-Wind, which is an unstructured-grid solver for the acoustically incompressible Navier-Stokes equations. The model consists of the mass-continuity Poisson-type equation for pressure and a momentum equation for the velocity. For such modeling approaches, simulation times are dominated by linear-system setup and solution for the continuity and momentum systems. For the ExaWind challenge problem, the moving meshes greatly affect overall solver costs because reinitialization of matrices and recomputation of preconditioners is required at every time step.

In this milestone report, we describe efforts to establish a body-resolved model of a megawatt-scale turbine that will be used for demonstrating and testing our wind farm simulation platform. The team chose the NREL 5-megawatt (MW) wind turbine, which is a notional turbine that is fully defined in the open domain. Given many proprietary issues surrounding modern turbines in the field (e.g., turbine control system, blade geometry and materials), a reference turbine such as the NREL 5-MW is ideal for the benchmarking and demonstration studies that will be key to the success of the ExaWind project. This milestone was designed to demonstrate a working model that will be used for figure-of-merit measurements and will establish baseline results that will be improved upon as the project progresses.

Two meshes, a 25 million node mesh and a 95 million node mesh, were generated using Pointwise meshing software that resolved the blades, nacelle, and tower. Rotor motion is handled through a sliding-mesh interface (developed earlier in the ExaWind project), for which the rotor and near-rotor fluid is embedded in a cylinder that rotates inside the larger, fixed domain. Two additional meshes were generated by uniformly refining the 95 million node mesh using Percept. Simulations using the coarsest mesh were run on the National Energy Research Scientific Computing Center (NERSC) Cori system under uniform inflow and with a wall-adapting local-eddy viscosity (WALE) turbulence model for approximately three rotations. Additionally, turbulent conditions (for inflow boundaries and volume initialization) were added using data from an external database of homogeneous isotropic turbulence (HIT). The HIT turbulence fields were derived from the Johns Hopkins Turbulence Database and turbulence intensity was scaled to $10 \%$, which is representative of atmospheric turbulence encountered by wind turbines in the field. Coarse-mesh simulations were also run with the generated HIT inflow and initial conditions.

The coarse-mesh WALE simulations with the sliding-mesh interface described here are a step toward predictive blade-resolved simulations that are the goal of ExaWind. The sliding-mesh interface is well suited for efficient simulations where the only turbine motion is that of the rotor. In operating turbines, individual blades rotate around their pitch axis, and the entire nacelle-rotor rotates (yaws) about the tower axis and translates because of tower deformations. Under these conditions, an overset-mesh approach is seen as necessary. It is understood that the WALE turbulence model with practical grid-refinement levels is insufficient for predictive simulations, and future simulations will, therefore, employ hybrid Reynolds-averaged Navier-Stokes (RANS)/large-eddy simulation (LES) turbulence modeling for which RANS will be employed near blade surfaces and LES will be employed in the farfield wake. Those simulations will also require much more refinement, especially near the blade surface. Finally, predictive simulations will need to be coupled to the OpenFAST turbine model, which models the nonlinear, largedeformation structural dynamics of the blades, the control system (that controls, e.g., pitch and yaw), and tower dynamics. These features not exercised in this milestone are all under development and will be demonstrated and described in the next ExaWind milestone report. 


\section{Acknowledgments}

This report was prepared as an account of work sponsored by an agency of the United States Government. Neither the United States Government nor any agency thereof, nor any of their employees, makes any warranty, express or implied, or assumes any legal liability or responsibility for the accuracy, completeness, or usefulness of any information, apparatus, product, or process disclosed, or represents that its use would not infringe privately owned rights. Reference herein to any specific commercial product, process, or service by trade name, trademark, manufacturer, or otherwise, does not necessarily constitute or imply its endorsement, recommendation, or favoring by the United States Government or any agency thereof. The views and opinions of authors expressed herein do not necessarily state or reflect those of the United States Government or any agency thereof.

This research was supported by the Exascale Computing Project (17-SC-20-SC), a joint project of the U.S. Department of Energy's Office of Science and National Nuclear Security Administration, responsible for delivering a capable exascale ecosystem, including software, applications, and hardware technology, to support the nation's exascale computing imperative.

This work was authored in part by the National Renewable Energy Laboratory, operated by Alliance for Sustainable Energy, LLC, for the U.S. Department of Energy (DOE) under Contract No. DE-AC36-08GO28308.

This research used resources of the National Energy Research Scientific Computing Center (NERSC), a U.S. Department of Energy Office of Science User Facility operated under Contract No. DE-AC02-05CH11231.

An award of computer time was provided by the Innovative and Novel Computational Impact on Theory and Experiment (INCITE) program. This research used resources of the Argonne Leadership Computing Facility, which is a DOE Office of Science User Facility supported under Contract DE-AC02-06CH11357. 


\section{Table of Contents}

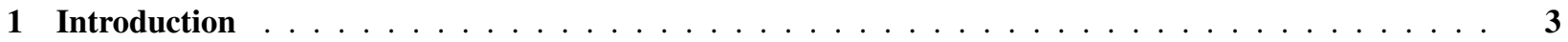

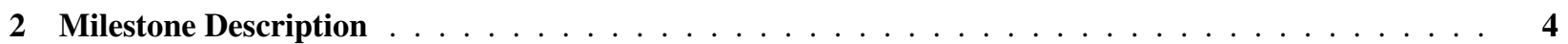

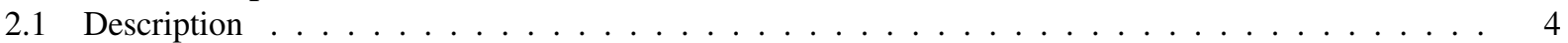

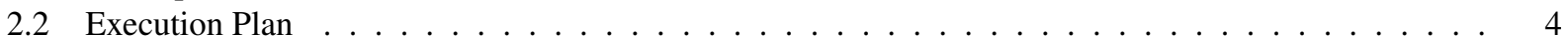

2.3 Overview of milestone completion $\ldots \ldots \ldots \ldots \ldots \ldots \ldots \ldots$

35 -MW NREL turbine model development $\ldots \ldots \ldots \ldots \ldots \ldots$

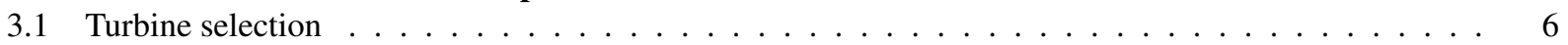

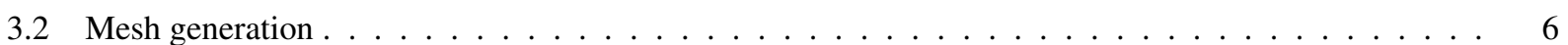

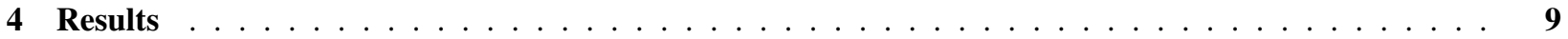

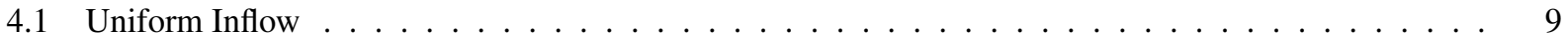

4.2 Turbulent Inflow $\ldots \ldots \ldots \ldots \ldots \ldots \ldots \ldots \ldots$

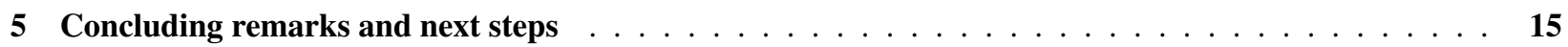

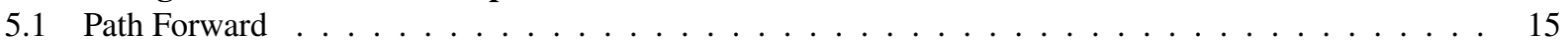

\section{List of Figures}

Figure 1. Image of the NREL 5-MW turbine g0 mesh showing the (upper left) domain extents, (lower left) rotor disk and wake refinement regions, (lower right) hub, nacelle, and blade mesh, and (upper right) blade tip mesh where the mesh extrusion off the blade surface is visible. . . . . . . . . . . . . . . .

Figure 2. Image of the NREL 5-MW turbine and its wake after the turbine has completed 2.3 revolutions at 14.0 seconds of simulation time. The wake is visualized by showing contours of velocity magnitude of

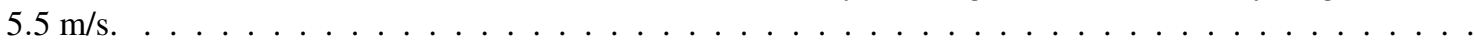

Figure 3. Initial conditions for the turbulent-inflow simulations. Top: Velocity magnitude is shown as a horizontal slice taken through the center of the hub. Bottom: Velocity magnitude is shown as vertical slice taken through the center of the turbine.

Figure 4. Velocity magnitude contours of $5.5 \mathrm{~m} / \mathrm{s}$ (blue) and $10.0 \mathrm{~m} / \mathrm{s}$ (red) after one revolution of the turbine. Left: Uniform initialization. Right: Turbulent initialization with $T I=0.1 \ldots \ldots \ldots$

Figure 5. The velocity magnitude is shown as a horizontal slice through the turbine hub with a 3D contour of $5.5 \mathrm{~m} / \mathrm{s}$ after one revolution of the turbine. Top: Uniform initialization. Bottom: Turbulent initialization with $T I=0.1 \ldots \ldots \ldots \ldots \ldots \ldots \ldots \ldots$ 


\section{List of Tables}

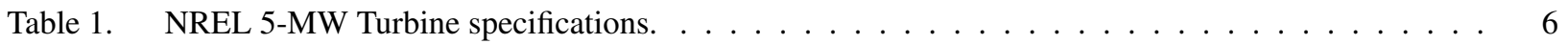

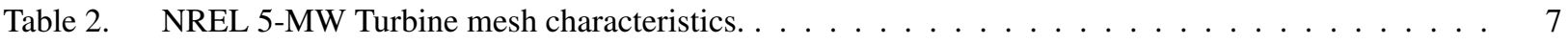




\section{Introduction}

The ultimate goal of the ExaWind project is to enable scientific discovery through predictive simulations of wind farms comprised of many megawatt-scale turbines situated in complex terrain. Predictive simulations will require computational fluid dynamics (CFD) simulations for which the mesh resolves the geometry of the turbines and captures the rotation and large deflections of blades. Whereas such simulations for a single turbine are arguably petascale class, multi-turbine wind farm simulations will require exascale-class resources.

The objective of this milestone is to demonstrate a "production run" of a megawatt-scale turbine to exercise and demonstrate the turbine simulation capabilities that have been developed during the previous two years of the ExaWind project. Demonstrating this simulation capability is the critical first step in enabling Nalu-Wind to be used as a predictive modeling tool that can advance the scientific understanding of wind turbine performance.

In Section 2 the milestone description is provided. Next, in Section 3, we present the NREL 5-megawatt (MW) reference turbine that was used to demonstrate Nalu-Wind's capabilities and describe the four computational meshes that were generated. In Section 4, we present results from simulations of the turbine operating under uniform inflow and homogeneous isotropic turbulence (HIT) conditions. Finally, in Section 5, notable achievements, conclusions, and a path forward are described. 


\section{Milestone Description}

In this section, we provide the approved milestone description and execution plan followed by a brief description of how the milestone was completed. Details regarding completion are included in the following sections.

\subsection{Description}

We will create and demonstrate a simulation capability for a megawatt-scale whole wind turbine operating in turbulent atmospheric flow where the fluid mesh resolves turbine geometry (blades, nacelle, tower). This model will include the key feature necessary for modeling a full wind farm comprised of many megawatt-scale turbines, including long, flexible blades.

Completion of this milestone will require integrating several modeling and simulation capabilities, including sliding or overset mesh capabilities to accommodate the rotating blades and mesoscale inflow conditions via a precursor atmospheric boundary layer (ABL) simulation or a numerical-weather-prediction code. A stretch goal will be to include fluid-structure-interaction coupling between the CFD code and the OpenFAST wind turbine code appropriate for flexible blades.

The OpenFAST turbine simulation tool includes nonlinear structural finite-element models and wind turbine controlsystem models. Several of these capabilities are being generated and verified in the U.S. Department of Energy's (DOE's) Office of Energy Efficiency and Renewable Energy's (EERE's) Atmosphere to Electrons (A2e) HighFidelity Modeling (HFM) project, which is running concurrently with this Exascale Computing Project (ECP).

\subsection{Execution Plan}

- Choose a baseline turbine configuration that has as many open source specifications as possible. If there is a lack of public-domain specifications, choose a reference turbine (e.g., the NREL 5-MW turbine, which is fully defined in the public domain).

- Create CFD models that resolve the baseline turbine outer geometry and turbine structural models in the OpenFAST code. Employ turbulence-modeling approaches demonstrated elsewhere in this project or in the A2e HFM project.

- Create inflow conditions representing relevant turbulent atmospheric flow.

- Create automated tests for full-turbine configuration.

- Perform simulations in relevant atmospheric conditions.

Completion Criteria: Technical report describing the milestone accomplishment as well as a highlight slide summarizing those accomplishments.

\subsection{Overview of milestone completion}

The following is a concise list of accomplishments that supported completion of this milestone:

- Chose the NREL 5-MW reference turbine as our baseline turbine, which is fully defined in the open domain.

- Created meshes at four refinement levels, ranging in size from 25-million nodes to 6-billion nodes, that resolve the blades, hub, nacelle, and tower. The meshes described in this report used the sliding-mesh interface developed earlier in the project (see, e.g., fiscal year FY 2018 Q1 milestone and Domino 2018). In ongoing work, an overset-mesh model is being created; the overset approach will be necessary for simulating an operating turbine with multiple moving and deforming parts.

- While the intention was to add a coarse-mesh model to the regression-test suite, the model sizes were too large (e.g., the coarsest mesh was $1.7 \mathrm{~GB}$ ). However, there is an existing regression test (dgNonConformalThreeBlade) that effectively tests the sliding-mesh interface used in this report. 
- Performed simulations on the NERSC Cori (Haswell) systems for the coarse mesh with a wall-adapting localeddy viscosity (WALE) turbulence model under uniform inflow and ran simulations for about three rotor revolutions.

- Performed simulations on the NERSC Cori (Haswell) systems for the coarse mesh (with a WALE turbulence model) using homogeneous isotropic turbulence (HIT) data for initialization and inflow boundary conditions. The HIT turbulence fields were derived from the the Johns Hopkins Turbulence Database and turbulence intensity was scaled to $10 \%$, which is appropriate for wind relevant flows. Simulations were run for about one full revolution.

- Demonstrated the ability to run the more refined meshes (up to 6 B nodes) on the Cori (Haswell) and Mira systems.

- Fluid-structure interaction capabilities for body-resolved meshes are under development and will be demonstrated and tested as part of a future milestone. 


\section{$3 \quad$ 5-MW NREL turbine model development}

\subsection{Turbine selection}

The NREL 5-MW reference turbine (Jonkman et al. 2009), with the specifications presented in Table 1, was selected to demonstrate Nalu-Wind's blade-resolved turbine simulation capabilities for the following reasons:

- It is fully open source and its design and performance characteristics are completely defined in Jonkman et al. (2009). This will allow the ExaWind team to share scientifically relevant results, as well as the meshes and input files, from the ExaWind project with the wider wind energy research and development (R\&D) community.

- It is widely used by the R\&D community to perform computational and experimental studies on a variety of topics, including but not limited to turbine wakes (e.g., Troldborg et al. (2012)), wind farm controls (e.g., Gebraad et al. (2017)), and offshore systems Lefebvre and Collu (2012). Accordingly, there are publicly available data sets from other research groups that can be compared to results generated with Nalu-Wind. In the future, the ExaWind and DOE HFM teams will be able to use the NREL 5-MW turbine model to study complex physical phenomena at an unprecedented level of detail with the goal of advancing wind turbine and wind farm technology.

- Fluid-structure interactions between the turbine blades and the atmospheric boundary layer (e.g., blade deformation and bent-twist coupling) affect the turbine performance. Therefore, the NREL 5-MW turbine provides an excellent test bed to demonstrate Nalu-Wind's fluid-structure interaction simulation capabilities.

- The turbine size is representative of a modern utility-scale wind turbine and simulations will enable a better understanding of the physics that govern turbine performance, directly benefiting the wind R\&D community.

\subsection{Mesh generation}

The first step in generating the mesh was developing a CAD model of the turbine using the information presented in Jonkman et al. (2009). The commercial meshing software Pointwise was then used to create two meshes: a coarse (gCoarse) mesh consisting of 25 million nodes and higher resolution mesh ( $\mathrm{g} 0$ ) consisting of 95 million nodes. The g0 mesh was then uniformly refined using Percept (part of the Trilinos software stack) to create g1 and g2 meshes. Table 2 presents the number of nodes and elements in each mesh, and Figure 1 illustrates the g0 mesh.

NREL 5-MW turbine volume meshes are fully unstructured, and consist of hexahedral, tetrahedral, pyramid, and wedge elements. The blade surface meshes are composed of structured quadrilateral elements. Hub, nacelle, and tower surface meshes are composed of unstructured quadrilateral-dominant meshes with very few triangular elements. A hexahedral-dominant boundary-layer mesh was extruded from all turbine surfaces (see Figure 1). The gCoarse mesh has a first cell height of $10^{-5} \mathrm{~m}$, and the g0 mesh has a first cell height of $10^{-6} \mathrm{~m}$. The growth rate for the boundary-layer meshes was 1.15 . The boundary-layer mesh for the gCoarse mesh ensured a $y+$ value of $\leq 100$

Table 1. NREL 5-MW Turbine specifications.

\begin{tabular}{|l|l|}
\hline Rating & $5 \mathrm{MW}$ \\
\hline Rotor Orientation, Configuration & Upwind, 3 Blades \\
\hline Control & Variable Speed, Collective Pitch \\
\hline Rotor Diameter & $126 \mathrm{~m}$ \\
\hline Hub Height & $90 \mathrm{~m}$ \\
\hline Cut-In, Rated, Cut-Out Wind Speed & $3 \mathrm{~m} / \mathrm{s}, 11.4 \mathrm{~m} / \mathrm{s}, 25 \mathrm{~m} / \mathrm{s}$ \\
\hline Cut-In, Rated Rotor Speed & $6.9 \mathrm{rpm}, 12.1 \mathrm{rpm}$ \\
\hline Rated Tip Speed & $80 \mathrm{~m} / \mathrm{s}$ \\
\hline Overhang, Shaft Tilt, Precone & $5 \mathrm{~m}, 5$ degrees, 2.5 degrees \\
\hline
\end{tabular}


Table 2. NREL 5-MW Turbine mesh characteristics.

\begin{tabular}{|l|l|l|}
\hline & Number of nodes (million) & Number of elements (million) \\
\hline gCoarse & 25 & 57 \\
\hline g0 & 95 & 176 \\
\hline g1 & 761 & 1415 \\
\hline g2 & 6082 & 11379 \\
\hline
\end{tabular}
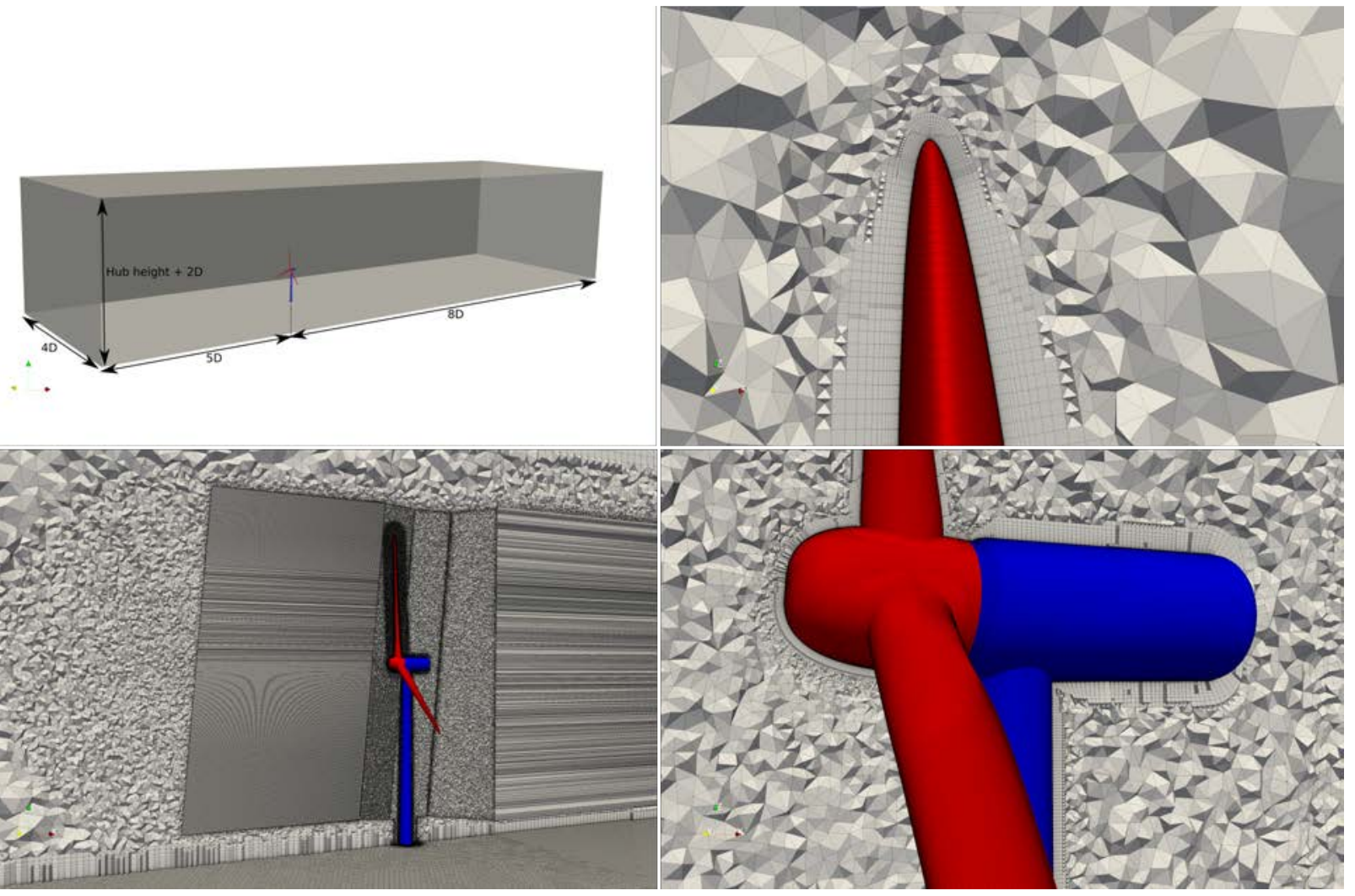

Figure 1. Image of the NREL 5-MW turbine g0 mesh showing the (upper left) domain extents, (lower left) rotor disk and wake refinement regions, (lower right) hub, nacelle, and blade mesh, and (upper right) blade tip mesh where the mesh extrusion off the blade surface is visible. 
for all cells, and the g0, g1, and g2 boundary-layer meshes ensured a $y+$ value $\leq 1$ for all cells when the turbine is operating at an oncoming flow velocity of $8.0 \mathrm{~m} / \mathrm{s}$. The remainder of the volume mesh consists of tetrahedraldominant isotropic elements.

The computational meshes are composed of two blocks, one containing the rotating blades and rotating portion of the hub, and another stationary domain that contains all nonrotating turbine components and the inflow and far-wake regions. This mesh topology enables the use of Nalu-Wind's nonconformal, discontinuous Galerkin (DG) capability as described in the FY18 Q1 ExaWind Report, to simulate the motion of the rotating mesh. 


\section{Results}

We performed two sets of simulations to demonstrate Nalu-Wind's simulation capability for a megawatt-scale turbine. First, we simulated the turbine in uniform flow in order to develop the preprocessing (i.e., mesh generation and refinement), simulation, and postprocessing methodologies on the ANL Mira and NERSC Cori systems. Next, we performed simulations of the turbine in a HIT inflow. The HIT simulations were performed by adding a turbulent velocity field to the mean velocity for both volume initialization and the inflow boundary condition. The turbulent velocity field was taken from external data of a forced homogeneous isotropic simulation run out to a statistically stationary state. Details on the uniform inflow simulations and the HIT turbulence simulations are described further in Sections 4.1 and 4.2.

All simulations were performed with the turbine operating in a flow with a mean velocity of $8.0 \mathrm{~m} / \mathrm{s}$. At this flow speed, the NREL 5-MW turbine is operating in "region 2," where the turbine runs at its design tip-speed ratio $V_{\text {blade-tip }} /|\mathbf{U}|=7.55$, where $V_{\text {blade-tip }}$ is the tip speed and $\mathbf{U}$ is the mean velocity, and a blade pitch angle of 0 degrees. At this operating point, the rotor rotational speed is 0.95 radians/second and it is operating at peak efficiency. Today's utility-scale turbines spend the majority of their operational lives running in "region 2"; therefore, the condition we have simulated is a highly relevant megawatt-scale turbine operating condition.

\subsection{Uniform Inflow}

The megawatt-scale simulation capability was demonstrated using the gCoarse mesh. Simulations were run on 26 Haswell nodes (832 cores) on the Cori system, with a distribution of 30,000 nodes per MPI rank. All uniform inflow simulations used the wall-adapting local eddy viscosity (WALE) LES model described in several previous ExaWind quarterly reports. Simulations were performed using the Trilinos linear-system solver stack (i.e., tpetra, MueLu, Belos, ifpack2) and the settings are described in Section 5 of the FY18 Q1 ExaWind report.

Seventeen seconds of simulation time, corresponding to 2.85 rotor revolutions, were completed. The simulation time step was adjusted so that the maximum Courant number was 100 across the entire computational domain, and after initial startup transients dissipated the time step converged to approximately $10^{-4}$ seconds. The 17 -second simulation required approximately 336 wall clock hours ( 14 wall clock days) to complete. Figure 2 presents a visualization of the simulation results. Rotor power and thrust were monitored during the simulation and, at 17 seconds of simulation time, these quantities were $1.9 \mathrm{MW}$ and $371 \mathrm{kN}$, respectively. These power and thrust values are within $6 \%$ and $20 \%$ of the steady-state values calculated using NREL's FAST reduced-order engineering model (Jonkman et al. 2009), respectively. While integrated quantities, such as power and thrust, calculated using engineering models and high-fidelity models typically show good agreement at steady state in uniform inflow, the discrepancies observed could be attributed to the following reasons: (1) The simulation has only completed approximately 3 rotor revolutions (it typically takes about 5-6 rotor revolutions for the power and thrust to converge to their quasi-steady values), and (2) The gCoarse mesh has a very coarse resolution of the blade boundary layer, thus it is expected that the viscous stresses are not adequately resolved for this mesh. The choice of gCoarse mesh was driven by practical considerations that required a tractable problem for debugging and testing out solver settings as well as developing the turbulent inflow capability.

We also performed uniform inflow situations on Cori using the g0, g1, and g2 meshes and on ALCF's Mira system using the $\mathrm{g} 0$ and $\mathrm{g} 1$ meshes in order to exercise the megawatt-scale turbine simulation capability on multiple platforms. g0, g1, and g2 simulations were all run for between 100 and 500 time steps and, in the future, these models can be used to update the figure of merit for the ExaWind project and to generate high-fidelity results that can be used to study turbine and wake physics at unprecedented levels of detail.

\subsection{Turbulent Inflow}

Our second test case focuses on establishing a capability to simulate the turbine under turbulent conditions. To accomplish this, we add a fluctuating field $\mathbf{u}^{\prime}(\mathbf{x}, t)$ of HIT, taken from external data, to both the initial velocity field 


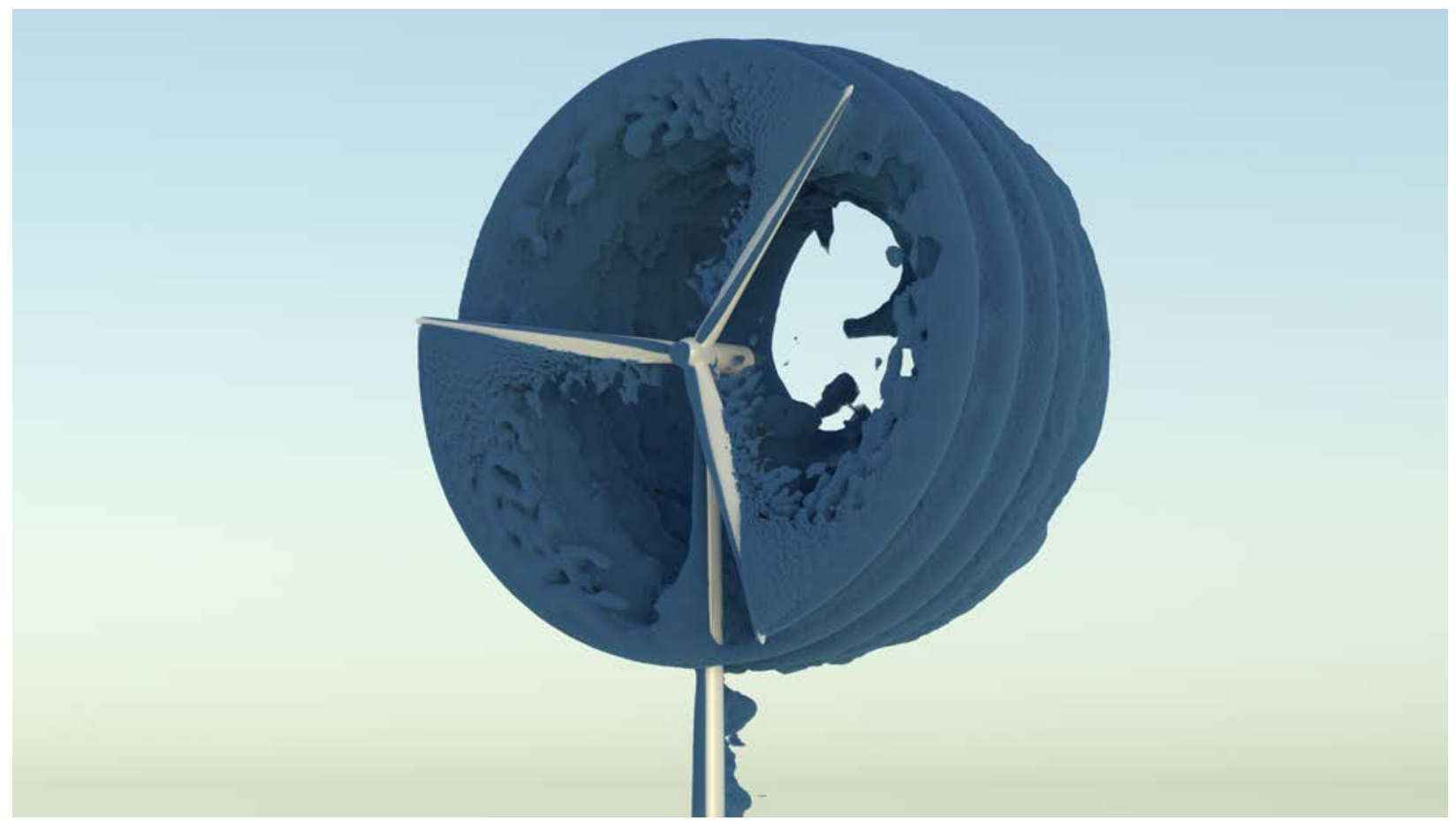

Figure 2. Image of the NREL 5-MW turbine and its wake after the turbine has completed 2.3 revolutions at 14.0 seconds of simulation time. The wake is visualized by showing contours of velocity magnitude of $5.5 \mathrm{~m} / \mathrm{s}$.

and the inflow condition at the inlet boundary. Using the same mean field $\mathbf{U}=(8 \mathrm{~m} / \mathrm{s}, 0,0)$ as in the uniform case described in Section 4.1, the initial condition for the turbulent inflow is defined as $\mathbf{V}(\mathbf{x}, t)=\mathbf{U}+\mathbf{u}^{\prime}(\mathbf{x}, t)$.

It must be noted that using HIT to represent the atmospheric boundary layer turbulence is a simplification as it does not capture the effect of atmospheric shear on the turbulent eddies. However, it is still a relevant step toward predictive simulations for the following reasons: (1) Synthetic HIT is the de facto standard for loads analysis during the design of wind turbine blades, and (2) Wind tunnel testing commonly uses grids that generate homogeneous isotropic turbulence, and many simulations employ synthetic HIT turbulence for studying wake dynamics (MartínezTossas et al. 2018; Kleusberg et al. 2017).

Following the approach described in Martínez-Tossas et al. (2018), we use the John's Hopkins Turbulence database http: / / turbulence.pha. jhu . edu / (JHTDB) to provide the fluctuating field $\mathbf{u}^{\prime}(\mathbf{x}, t)$. The JHTDB HIT case is a $1024^{3}$ triply periodic $(2 \pi)^{3}$ box forced to a statistically stationary state. Once it reached the stationary state, the velocity field was extracted and stored with over 5000 time steps covering approximately five large-eddy turnover times ( $\mathrm{Li}$ et al. 2008). This extensive range of data provides a more-than-sufficient tool to create a $\mathbf{u}^{\prime}(\mathbf{x}, t)$ field covering the whole domain and an inflow condition over time. Another benefit of the JHTDB is it provides an easy-to-access web API that can be interfaced with a $\mathrm{C}++$ code to generate the initial and inflow exodus files for our Nalu-Wind turbulent inflow simulations. The getBoxFilter() function in the database was used with a specified filter width chosen to match the grid resolution in the mesh onto which the database was mapped, which provides the added benefit of filtering the HIT data to match the resolution allowed by the physical simulation.

To generate the $\mathbf{u}^{\prime}(\mathbf{x}, t)$ field from the HIT data, we start by defining a desired turbulent intensity $T I$, which is then used to impart a mean velocity of $\mathbf{U}_{H I T}=\mathbf{u}_{R M S} / T I$ onto the database, where $\mathbf{u}_{R M S}$ is the specified root-mean-square turbulent velocity observed in the database. This defines the velocity scale for the HIT data, whereas the velocity scale for our physical simulation is the underlying mean flow $(8 \mathrm{~m} / \mathrm{s})$. Next, we define the length scales, which come naturally. Our HIT data has a length scale from the box size of $L_{H I T}=2 \pi$ which will need to be stretched to match 
the length scale in our turbine simulation. Since our turbine domain is rectangular in the $\mathrm{y}-\mathrm{z}$ plane $(4 D \times 2.71 D$, where $D$ is the turbine-rotor diameter), we take the larger of the two dimensions as our physical length scale, $L=4 D$. With a length and velocity scale defined, we can then define timescales $T_{H I T}=L_{H I T} /\left|\mathbf{U}_{H I T}\right|, T=L /|\mathbf{U}|$, for both the database and physical simulation, respectively.

Next, an initial starting time $t_{0}$ and location $x_{0}$ are chosen in the database. The velocities in the $y-z$ plane at $\left(x_{0}, t_{0}\right)$ are extracted from the database and stretched by the length scale ratio $L / L_{H I T}$ to represent the initial field at the inflow boundary $x=-5 D \mathrm{~m}$ in the physical domain. Since $L$ is taken to be the larger of the $y$ and $z$ extents, this implies that only a subset of the full $y-z$ field is mapped onto the physical domain. This method was chosen to prevent stretching the turbulent field in an anisotropic way. The velocities from the database are scaled by the velocity scale ratio $|\mathbf{U}| /|\mathbf{U}|_{H I T}$ to ensure that the desired $T I$ specified in the first step is met. To initialize the $y-z$ plane at any other $x$ location in the physical domain, the amount of time needed for the flow to reach that point from the inflow boundary is calculated as $t=(x+5 D) /|\mathbf{U}|$. This is then scaled by the ratio of timescales to the corresponding database time, $t_{H I T}=t * T_{H I T} / T$. With the associated $t_{H I T}$, the $y-z$ plane in the database at $\left(x_{0}+(x+5 D) * L_{H I T} / L, t_{0}+t_{H I T}\right)$ is selected. Taking advantage of the periodic nature of the flow in the database, locations in the database mapped to points outside of the $2 \pi$ boundaries are represented by their corresponding periodic location inside the domain. This allows us to create an entire initial condition that represents the evolution of a single slice of the HIT domain, stretched to fit our physical domain and scaled to represent the desired turbulent intensity $T I$. The same process can be used to generate the inflow boundary condition, where the $x$ location is chosen to be at some point upstream of the inflow boundary, associated with some physical time $t$ that would have to elapse before that point reaches the inflow plane.

Because of the complicated mesh of the physical domain, and to leverage the built-in solution transfer capability provided by Nalu-Wind, the volume initialization and inflow conditions are stored on an intermediate mesh that has the same domain as the physical mesh, but has only 128 evenly spaced gridpoints in the $y$ direction, with $d x$ and $d z$ chosen to be approximately equal to the resulting $d y$. The initial condition and inflow conditions are then linearly interpolated onto the actual turbine mesh as part of the external data transfer routine in Nalu-Wind.

In Figure 3, the initial field with the added synthetic HIT is shown for both a horizontal slice through the center of the hub (left) and a vertical slice at the center of the turbine (right). Qualitatively, there do not appear to be any negative impacts on the structures from the interpolation, and it can be seen that the turbine will experience turbulent conditions upon startup of the simulation.

The turbulent inflow simulation was run for a single revolution of the turbine $(\approx 6.6 \mathrm{~s})$ and then compared to the uniform inflow simulation described above. Since this is not a sufficient time to measure quantitative values, we rely on qualitative comparisons between the two runs. In Figure 4, we show the velocity magnitude contours of $5.5 \mathrm{~m} / \mathrm{s}$ (blue) and $10.0 \mathrm{~m} / \mathrm{s}$ (red). The left plots are from the uniform initialization simulation and the right plots are from the turbulent initialization with $T I=0.1$. Figure 5 shows the velocity magnitude as a horizontal slice through the turbine hub with a $3 \mathrm{D}$ contour of $5.5 \mathrm{~m} / \mathrm{s}$. The top plot shows uniform initialization and the bottom plot shows turbulent initialization with $T I=0.1$. Although the simulation has only completed a single revolution under turbulent inflow, it demonstrates the ability to perform blade-resolved simulations under turbulent inflow conditions with Nalu-Wind.

As expected, a comparison of the uniform inflow and HIT turbulence inflow simulations shows a much more symmetric structure for the uniform case. The turbulent case has a more complex and chaotic wake structure that breaks down earlier than the wake of the uniform inflow case because of the influence of the HIT turbulence. We note that with the selected contours of $5.5 \mathrm{~m} / \mathrm{s}$ and $10.0 \mathrm{~m} / \mathrm{s}$, we are just at the edge of the tails for the velocity distribution after adding in the external HIT field. This allows for the clean view of the turbine and the wake in the turbulent flow cases shown here.

Additional work related to the turbulent inflow cases will be focused on adding shear to the turbulence to allow for a more accurate representation of atmospheric turbulence as well as comparing the turbulent flow cases generated here to those where turbulence from a precursor ABL simulation is used. In addition, we are observing some spurious oscillations originating from the inflow boundary and propagating along the terrain boundary, which we are currently investigating. 

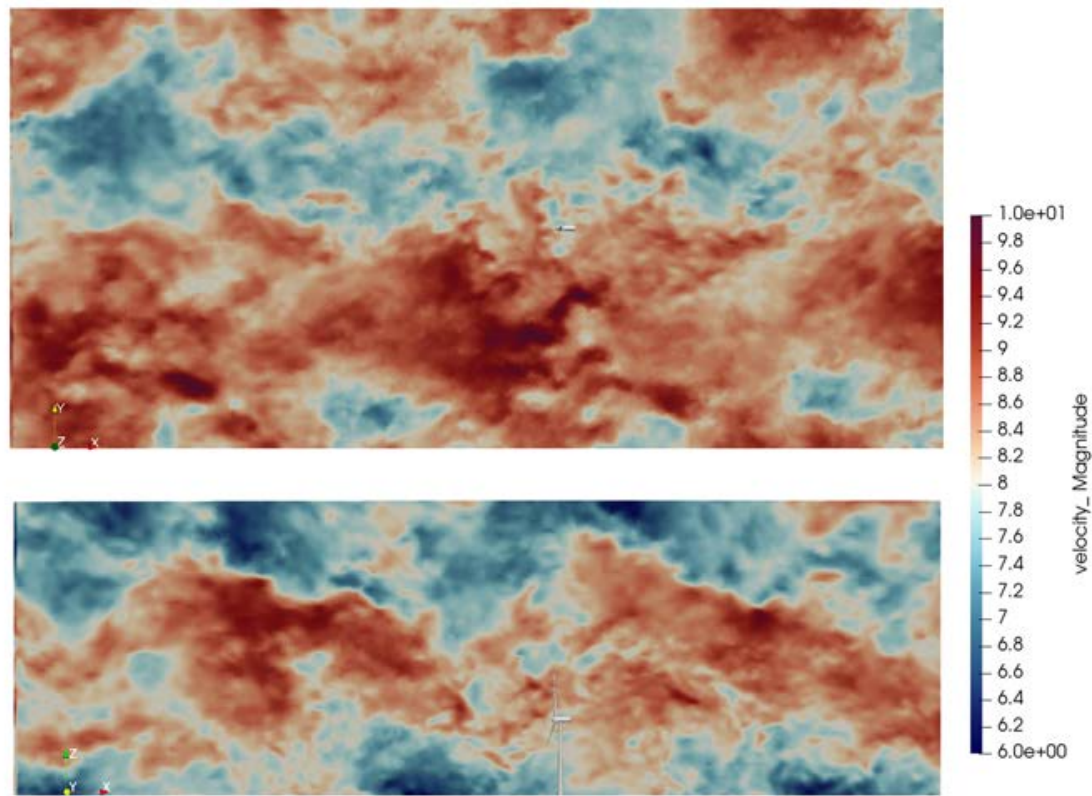

Figure 3. Initial conditions for the turbulent-inflow simulations. Top: Velocity magnitude is shown as a horizontal slice taken through the center of the hub. Bottom: Velocity magnitude is shown as vertical slice taken through the center of the turbine. 

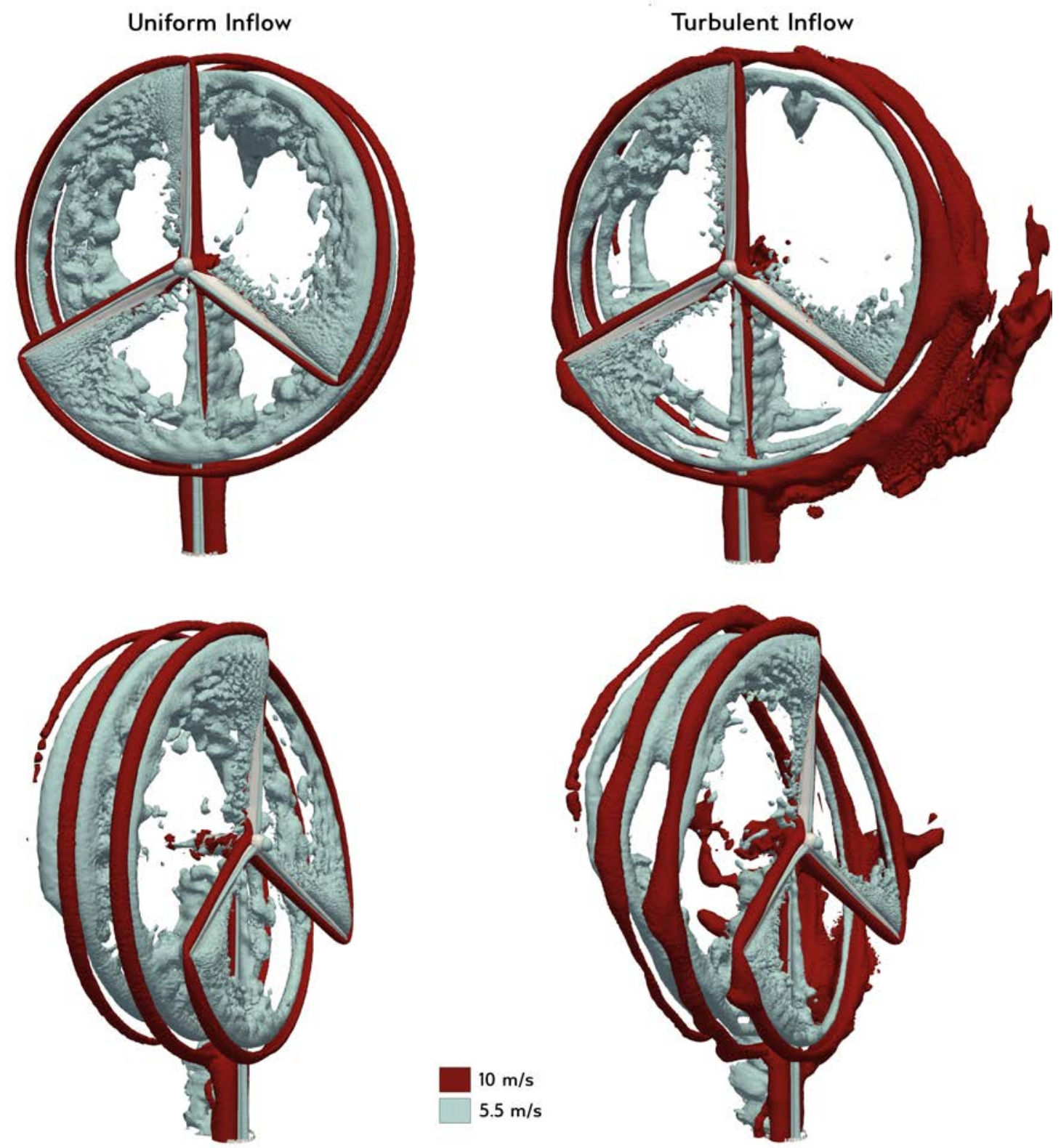

Figure 4. Velocity magnitude contours of $5.5 \mathrm{~m} / \mathrm{s}$ (blue) and $10.0 \mathrm{~m} / \mathrm{s}$ (red) after one revolution of the turbine. Left: Uniform initialization. Right: Turbulent initialization with $T I=0.1$. 

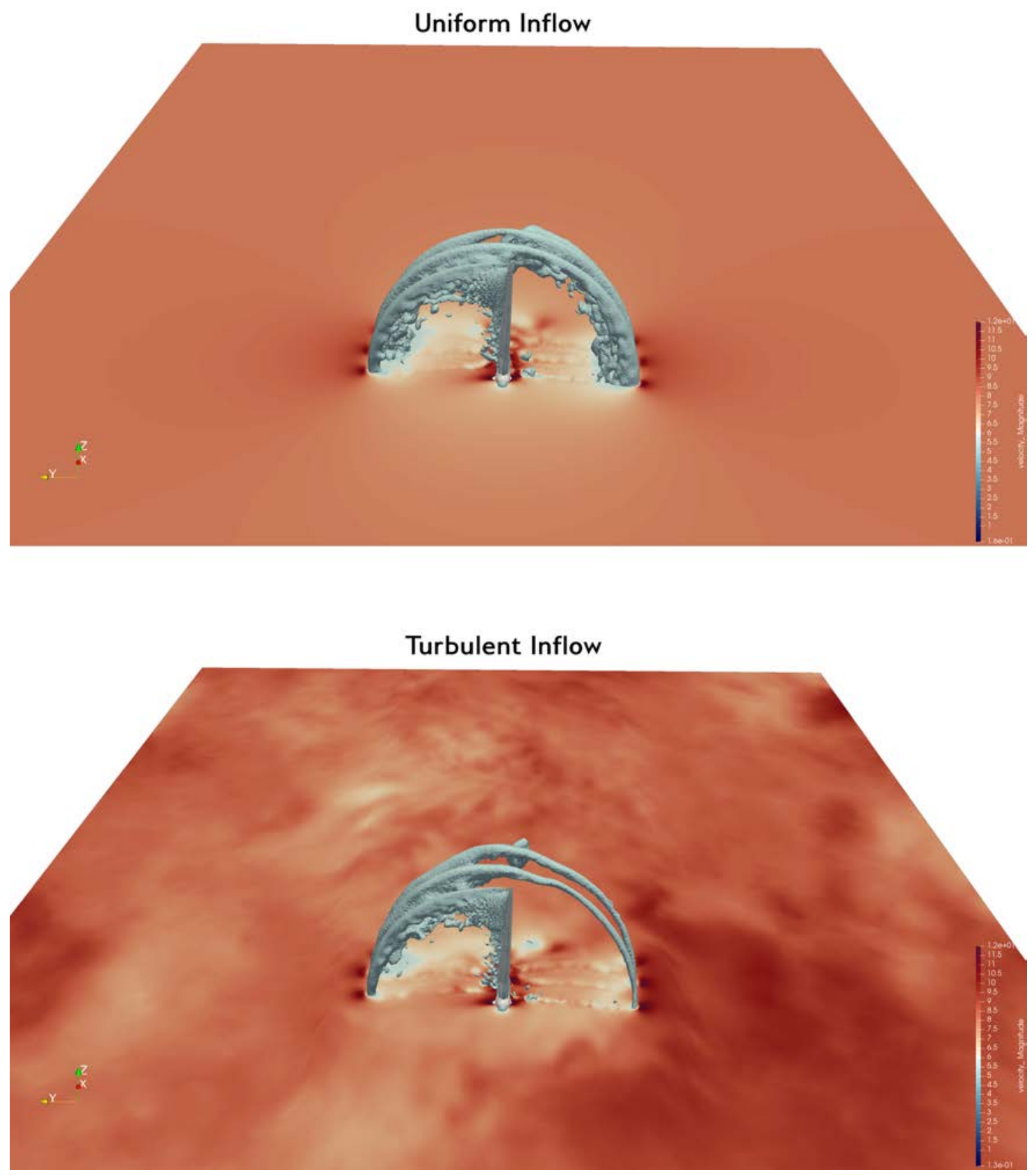

Figure 5. The velocity magnitude is shown as a horizontal slice through the turbine hub with a 3D contour of $5.5 \mathrm{~m} / \mathrm{s}$ after one revolution of the turbine. Top: Uniform initialization. Bottom: Turbulent initialization with $T I=0.1$. 


\section{Concluding remarks and next steps}

In this milestone effort, we developed and demonstrated a megawatt-scale turbine simulation capability. The NREL 5-MW reference turbine was selected to demonstrate the capability because it is fully open source and widely used by the wind turbine R\&D community. Other reasons for selecting the NREL 5-MW reference turbine are discussed in Section 3. Four grids (gCoarse: 25 million nodes, g1: 95 million nodes, g1: 761 million nodes, and g2: 6.082 billion nodes) were generated and used to exercise Nalu-Wind's per-processing, simulation, and post-processing capabilities on the Cori and Mira systems by running simulations between 50 and 500 timesteps. We used the gCoarse mesh (25-M nodes) to perform a production simulation of the NREL 5-MW turbine in both uniform inflow and HIT synthetic turbulence inflow. Approximately 3 turbine revolutions were simulated under uniform inflow and 1 turbine revolution was simulated under HIT conditions. Although the gCoarse simulations have not yet reached steady state, the uniform inflow simulation power and thrust values are within $6 \%$ and $20 \%$, respectively, of the expected values that were calculated using NREL's FAST reduced-order turbine simulation tool (Jonkman et al. 2009).

A comparison of the wake structures between the uniform inflow and HIT synthetic turbulence inflow simulations shows that wake structures are significantly affected by the presence of turbulence. Specifically, the results pre-

sented in Figure 4 show that, as expected, the wake structure is more irregular and breaks down faster under turbulent conditions. Using Nalu-Wind's ability to model wake propagation and breakdown under atmospheric conditions, simulations performed using the more resolved $\mathrm{g} 0 \mathrm{~g} 2$ meshes will provide the wind R\&D community with an unprecedented level of understanding of turbine and wind farm physics that is required to develop the next generation of wind turbine and farm technologies.

\subsection{Path Forward}

The ExaWind team will explore the following paths forward to further develop the Nalu-Wind megawatt-scale turbine simulation capability and to work toward FY19 ExaWind milestones:

- Run the gCoarse mesh under uniform inflow and HIT synthetic turbulence inflow to steady state to develop baseline simulation characteristics (e.g., how long it takes integrated quantities, such as power and thrust, to come to steady state).

- Simulate the NREL 5-MW turbine using the g0 mesh with the k-omega SST-DES model (as developed for the FY17 Q3 ExaWind milestone) under uniform inflow and atmospheric turbulence conditions. Use the results to compare Nalu-Wind results to publicly available NREL-5 MW reference turbine performance data to better understand the physics of turbine performance.

- Perform strong and weak scaling studies on the Mira and Cori systems to understand how Nalu-Wind's solvers scale on meshes up to 6 billion nodes.

- Update the ExaWind figure of merit using the g1 mesh on Cori and the g2 mesh on Mira.

- Create a blade-resolved wind farm model by stacking several gCoarse or g0 meshes. Use this mesh to demonstrate Nalu-Wind's wind farm simulation capabilities and to perform scaling studies using meshes with several sliding mesh interfaces. 


\section{Bibliography}

Domino, S.P. 2018. "Design-order, non-conformal low-Mach fluid algorithms using a hybrid CVFEM/DG approach". Journal of Computational Physics 349:331-351.

Gebraad, P., et al. 2017. "Maximization of the annual energy production of wind power plants by optimization of layout and yaw-based wake control". Wind Energy 20 (1): 97-107.

Jonkman, J., et al. 2009. "Definition of a 5-MW reference wind turbine for offshore system development". National Renewable Energy Laboratory, Golden, CO, Technical Report No. NREL/TP-500-38060.

Kleusberg, E., et al. 2017. "High-Order Numerical Simulations of Wind Turbine Wakes". Journal of Physics: Conference Series 854:012025.

Lefebvre, S., and M. Collu. 2012. "Preliminary design of a floating support structure for a 5 MW offshore wind turbine". Ocean Engineering 40:15-26.

$\mathrm{Li}$, Y., et al. 2008. "A public turbulence database cluster and applications to study Lagrangian evolution of velocity increments in turbulence". Journal of Turbulence 9:N31.

Martínez-Tossas, L.A., et al. 2018. "Comparison of four large-eddy simulation research codes and effects of model coefficient and inflow turbulence in actuator-line-based wind turbine modeling". Journal of Renewable and Sustainable Energy 10:033301.

Troldborg, N., et al. 2012. "Comparison of the wake of different types of wind turbine CFD models". In 50th AIAA Aerospace Sciences Meeting including the New Horizons Forum and Aerospace Exposition, 237. 